\title{
Automatic Extraction of Femur Contours from Calibrated Fluoroscopic Images
}

\author{
Xiao Dong Miguel A. Gonzalez Ballester Guoyan Zheng \\ University of Bern, MEM Research Center \\ Stauffacherstrasse 78, 3014-Bern, Switzerland \\ Guoyan.Zheng@MEMcenter.unibe.ch
}

\begin{abstract}
Automatic identification and extraction of bone contours from $x$-ray images is an essential first step task for further medical image analysis. In this paper we propose a $3 D$ statistical model based framework for the proximal femur contour extraction from calibrated $x$-ray images. The automatic initialization is solved by an Estimation of Bayesian Network Algorithm to fit a multiple component geometrical model to the $x$-ray data. The contour extraction is accomplished by a non-rigid $2 D / 3 D$ registration between a $3 D$ statistical model and the $x$-ray images, in which bone contours are extracted by a graphical model based Bayesian inference. Preliminary experiments on clinical data sets verified its validity.
\end{abstract}

\section{Motivation}

Fluoroscopic images are still playing a crucial role in diagnosis and surgery. Accurate detection and extraction of bone contours from fluoroscopic images is an essential component for computer analysis of medical images for diagnosis [1][2][3], planning [4][5][6] or 3D reconstruction of anatomic structures [7][8][9][10]. Fluoroscopic images can vary a lot in terms of brightness and contrast as well as in the imaged region of anatomy. Therefore conventional segmentation techniques [1][5][6] can not offer a satisfactory solution and model based segmentation is usually implemented to obtain robust and accurate results [3][7][11][12][13].

In [3][12][14][13][15], 2D statistical models, Adaptive Shape Model (ASM) or Adapative Appearance Model (ASM), are constructed from a training image set under the assumption that the images are taken from a certain view direction. 2D statistical models can contain both the shape and texture information learnt from training data set, which is helpful in improving robustness and accuracy in noisy images. 2D statistical model asks for a proper initialization due to the limited convergence region. Fully automatic ini- tialization can be accomplished by the generalized Hough transformation [12], neural nets [13] or evolutionary algorithms [14][15]. But both the initialization and segmentation performance relies on that the view direction assumption can be fulfilled.

In [7][8][9][10] 3D statistical models are used for 2D segmentation and 3D reconstruction from calibrated 2D fluoroscopic images (location and orientation of the fluoroscopic source w.r.t. the image acquisition planes are known). Compared with 2D statistical modes, 3D statistical model usually only contains shape information but not the intensity information on the 2D images. In principle it can be used for segmenting an image taken from an arbitary view direction. 3D statistical model also need an initialization, which is usually manually defined [7][9]. Due to the dense mesh of the 3D statistical model [16], fully automated solutions based on evolutionary algorithm is computational expensive [17].

In this paper we propose a 3D statistical model based fully automatic segmentation framework for calibrated fluoroscopic images. In our approach, the initialization is accomplished by an Estimation of Bayesian Network Algorithm on a simplified multiple component model instead of the triangulated surface mesh of the 3D model, which reduces the computational complexity. The statistical model based fine shape extraction is achieved by a Bayesian inference on a Bayesian network, which encodes the shape and texture information of the model and therefore enhances the robustness and accuracy of the contour extraction.

\section{Related Work}

Bayesian network based approach [18][19][20] is used to identify or track object such as human body, which is composed with multiple subparts and among the subparts structral or kinematic constrains exist. The Bayesian network embeds the subparts constraints in a graphical model associated with image observations. Bayesian network is also exploited for finding deformable shapes [21][22], where both 
the local and relative shape information can be encoded in a graphical model and a Bayesian inference is carried out to find the solution.

\section{Methods}

\subsection{Image acquisition}

We use calibrated fluoroscopic images from C-arm. Due to the limited imaging volume of C-arm, we ask for four images for the proximal femur from different view direction, of which two images focus on the proximal femoral head and the other two focus on the femoral shaft. The calibrated fluoroscopic image set is represented by $\mathbf{I}$.

\subsection{Statistical model of the proximal femur}

A Principle Component Analysis (PCA) based 3D statistical model $\mathcal{M}$ with 4098 vertices of the proximal femur is constructed from a training data set containing the CT data of 13 bones [16] as shown in Fig. 1(a). An instance generated from the statistical model with parameter set $\mathbf{Q}=\left\{\alpha, \beta_{0}, \beta_{1}, \ldots, \beta_{11}\right\}$ can be described as

$$
\mathcal{M}: \mathbf{S}(\mathbf{Q})=\alpha\left(\mathbf{S}_{0}+\sum_{i=0}^{11} \beta_{i} \lambda_{i}^{\frac{1}{2}} \mathbf{P}_{i}\right)
$$

where $\mathbf{S}_{0}$ is the mean model, $\alpha$ is the scaling factor, $\lambda_{i}$ and $\mathbf{P}_{i}$ are the $i$ th eigenvalue and the the correspondent eigenvectror.

\subsection{Automated initialization}

To find the initial rigid transformation $\mathbf{T}_{0}$ and parameter set $\mathbf{Q}_{0}$ to align the model instance $\mathbf{S}\left(\mathbf{Q}_{0}\right)$ with the observed fluoroscopic images, a multiple component geometrical model is constructed for the proximal femur. A Bayesian network is established to encode the constraints among the components and an Estimation of Bayesian Network Algorithm (EBNA) is used to align the geometrical model with the fluoroscopic images. Then $\mathbf{T}_{0}$ and $\mathbf{Q}_{0}$ can be calculated from the geometrical model accordingly.

\subsubsection{Multiple component model of the proximal fe- mur}

The proximal femur is modeled by a geometrical model consisting of 3 components: head, neck and shaft, which are described by a sphere, a trunked cone and a cylinder with parameter set $\mathbf{X}_{g e o}=\left\{\mathbf{X}_{H}, \mathbf{X}_{N}, \mathbf{X}_{S}\right\}$ respectively as shown in Fig. 1(b). On one hand the three components are constrained by the anatomical structure of the proximal femur. Compared with 2D model based initialization

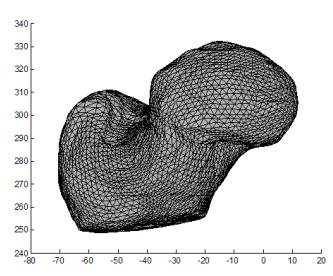

(a) PCA based 3D statistical model

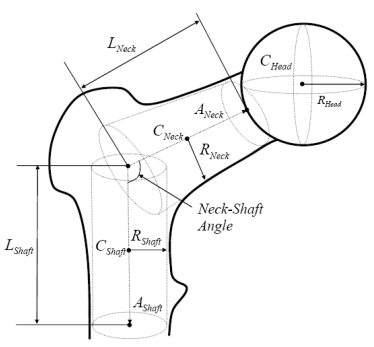

(b) Multiple component geometrical model
Figure 1. 3D models of the proximal femur for automatic femur contour extraction

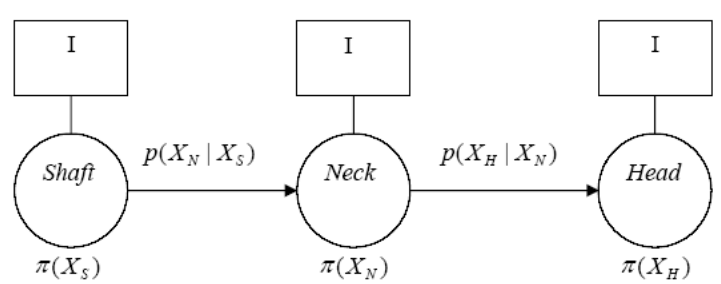

Figure 2. Graphical model for the multiple component geometrical model fitting, $\pi(\cdot) \mathbf{s}$ are the prior distributions of nodes and $p(\cdot \cdot \cdot) \mathbf{s}$ are the conditional distributions, $I$ is the observed image data

[1][12][15], the simplified 3D model is less view direction dependent and has the capability to catch the global structure of the anatomy from the fluoroscopic images. On the other hand the simplified geometrical model is much less computational expensive than the statistical model with dense surface mesh [17].

\subsubsection{Bayesian network for the proximal femur model}

The constraints among components are encoded in the conditional distributions among the nodes in a graphical model [18][20][23] as shown in Fig. 2. The conditional distributions are set so that the geometrical model can represent a meaningful anatomical structure of the proximal femur.

\subsubsection{Geometrical model fitting by EBNA}

A combination of particle filter and probability logic sampling, which can also be regarded as an EBNA is implemented to fit the geometrical model with the fluoroscopic images by solving the maximal likelihood estimation

$$
\left(\mathbf{X}_{g e o}^{*}\right)=\max _{\mathbf{X}_{g e o}} \operatorname{Prob}\left(I \mid \mathbf{X}_{g e o}\right)
$$


where $\operatorname{Prob}\left(I \mid \mathbf{X}_{\text {geo }}\right)$ is called observation model. It's constructed by a similarity measurement between the fluoroscopic images and the silhouettes of the projected geometrical model on the correspondent image planes, which is calculated as the AAM. The EBNA is given in Algorithm 1.

1. Initialization
Generate the first generation of particle set with $N$
particles $\left\{P_{i}^{0}=\mathbf{X}_{\text {geo }, i}^{0}\right\}_{i=0, \ldots, N-1}$ from the proposal
distributions
$q^{0}\left(\mathbf{X}_{S}\right)=\pi\left(\mathbf{X}_{S}\right)$
$q^{0}\left(\mathbf{X}_{N}\right)=\pi\left(\mathbf{X}_{N}\right) q^{0}\left(\mathbf{X}_{S}\right) p\left(\mathbf{X}_{N} \mid \mathbf{X}_{S}\right)$
$q^{0}\left(\mathbf{X}_{H}\right)=\pi\left(\mathbf{X}_{H}\right) q^{0}\left(\mathbf{X}_{S}\right) p\left(\mathbf{X}_{H} \mid \mathbf{X}_{N}\right)$
2. Observation
Given the current generation of particle set, calculate
the weight of each particle as $w_{i}^{n} \propto$ Prob $\left(I \mid \mathbf{X}_{\text {geo }, i}^{n}\right)$
3. Update
Update the proposal distributions as
$q^{n+1}\left(\mathbf{X}_{S}\right)=N P D E\left(w_{i}^{n}, \mathbf{X}_{S, i}^{n}\right)$
$q^{n+1}\left(\mathbf{X}_{N}\right)=\pi\left(\mathbf{X}_{N}\right) q^{n+1}\left(\mathbf{X}_{S}\right) p\left(\mathbf{X}_{N} \mid \mathbf{X}_{S}\right)$
$q^{n+1}\left(\mathbf{X}_{H}\right)=\pi\left(\mathbf{X}_{H}\right) q^{n+1}\left(\mathbf{X}_{N}\right) p\left(\mathbf{X}_{H} \mid \mathbf{X}_{N}\right)$
where $N P D E\left(w_{i}^{n}, \mathbf{X}_{S, i}^{n}\right)$ is a nonparametric density
estimation. Generate the next generation of particle
set from the updated proposal distributions.
4. Go to 2 until the particle set converges.

Algorithm 1. EBNE for geometrical model fitting

\subsubsection{Statistical model initialization}

From the mean shape of the $3 \mathrm{D}$ statistical model $\mathbf{S}_{0}$, the model vertices can be classified into three regions, femoral head, neck and shaft. The femoral head center and radius, axes of femoral neck and shaft can be determined in the model coordinate space by a 3D sphere fitting to the femoral head region and cylinder fittings to the femoral neck and shaft regions. The initial rigid transformation and scale can then be computed to fit the statistical model (the scaled mean shape) to the geometrical model.

\subsection{D statistical model based contour ex- traction}

After the statistical model initialization, the contour extraction is accomplished by a joint registration and segmentation as summarized in Algorithm 2.

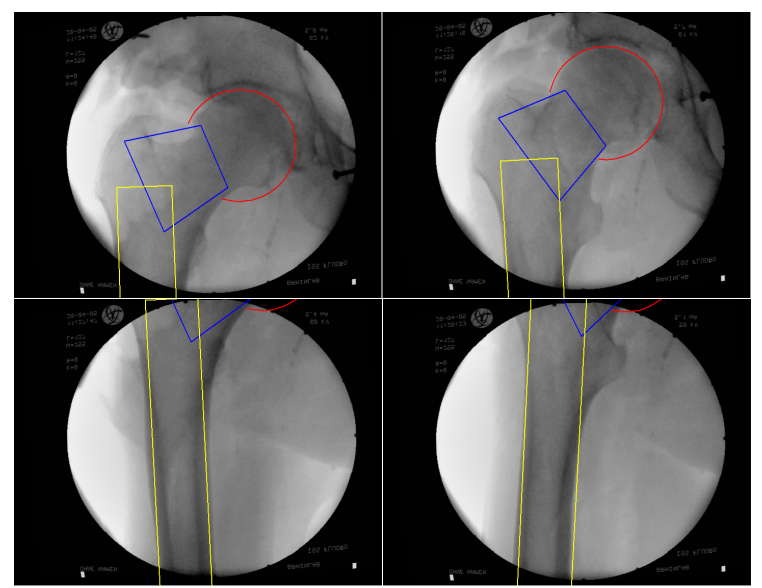

(a) Fitting the geometrical 3D model with fluoroscopic images

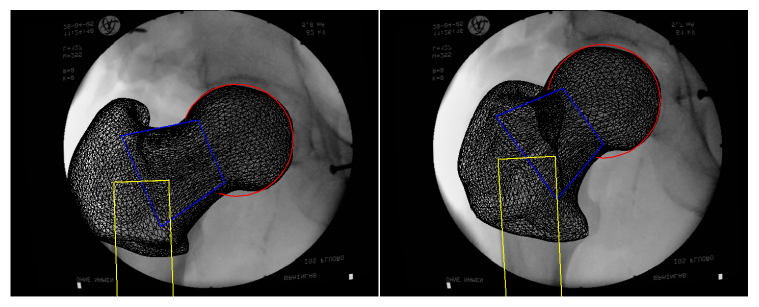

(b) Fitting the statistical model with the geometrical model

Figure 3. Automatic 3D statistical model initialization 
1. Simulated fluoroscopic and silhouette extraction Given the current instanced statistical model $\mathcal{M}: \mathbf{S}\left(\mathbf{Q}^{n}\right)$ and the transformation $\mathbf{T}^{n}$ to align the 3D model to the observed fluoroscopic images, project the aligned statistical model on each of the $K$ fluoroscopic image planes using the projection geometry of each fluoroscopic image. From the simulated fluoroscopic images the silhouettes $\left\{\mathbf{C}_{\text {model }}^{k, n}\right\}_{k=0, \ldots, K-1}$ are extracted [8].

\section{2. $2 D$ template based segmentation}

On each fluoroscopic image, taking the correspondent silhouette of the projected statistical model $\mathbf{C}_{\text {model }}^{k, n}$ as a template, a Bayesian network based shape matching is implemented to search for the bone contour $\mathbf{C}_{\text {image }}^{k, n}$.

\section{Nonrigid $2 D / 3 D$ registration}

A 2D/3D nonrigid registration procedure as described in [8] is carried out to fit the extracted bone contours $\mathbf{C}_{\text {image }_{k=0, \ldots, K-1}^{k, n}}$ and the statistical model $\mathcal{M}$, which results in an updated instanced model $\mathcal{M}: \mathbf{S}\left(\mathbf{Q}^{n+1}\right)$ and rigid transformation $\mathbf{T}^{n+1}$

4. Go to 1 , until the procedure converges.

Algorithm 2. Statistical model based segmentation

\subsubsection{D template based segmentation using belief propagation}

Usually active contour or statistical model (ASM or AAM) [1][11] are used for model based 2D segmentation. Ideally the model should keep the global shape information as well as involve the local feather information such as the edge and intensity distribution. Active contour emphasizes on searching for local features along the contour and therefore usually lacks of the ability to hold the global structure. ASM/AAM consider both the global shape and the local features. But its shortcoming is that its searching strategy usually independently estimate each point to a new target position by a local search along the normal direction of the contour. Therefore the inaccuracies in this estimation can not be appropriately accommodated for by the global shape information and can only be regularized by a projection to the shape space during the nonrigid registration step [8]. In [21][22] the shape matching problem is formalized as an Bayesian inference on a graphical model and solved by loopy belief propagation and Bathe free energy approximation respectively. In this approach, a graphical model is established so that the correspondence assignment for each point involves both the global shape and local feature information.

From the silhouette of the projected 3D statistical model, we sample $M$ points(nodes) tracing the contour as the shape prior. Each point is described by a parameter set $\mathbf{q}_{i}=\left\{\mathbf{x}_{i}, \mathbf{g}_{i}\right\}, i=0, \ldots, M-1$, where $\mathbf{x}_{i}=\left(x_{i}, y_{i}\right)$ is the position of $i$ th point on the image coordinate system, $\mathbf{g}_{i}=\left(g x_{i}, g y_{i}\right)$ is the image gradient of the current node. Due to the lack of 2D training data set, we define that $\left\|g_{i}\right\|=1$ and its direction is parallel with the local direction of the contour on the normalized gradient image. The configuration of our model can then be written as $\mathbf{Q}_{\text {model }}=\left\{\mathbf{q}_{i}\right\}_{i=0, \ldots, M-1}$ and the configuration of a candidate contour can be written as $\mathbf{Q}_{\text {cand }}=\left\{\mathbf{q}_{i}^{\prime}\right\}_{i=0, \ldots, M-1}$.

We then establish a partially connected graph with $M$ vertices as: $\mathbf{G}(\mathbf{V}, \mathbf{E}), \mathbf{V}=\left\{v_{i},\right\}_{i=0, \ldots, M-1}, \mathbf{E}=$ $\left\{e_{i, j}\right\}_{i, j=0, \ldots, M-1}$, where $e_{i, j}=1$ for $(i-j) \bmod M<$ $4, i \neq j$, i.e., each vertex is connected with its 6 nearest neighbours. We define the potential between vertices $i$ and $j$ with $e_{i, j}=1$ as

$$
U_{i, j}\left(\mathbf{q}_{i}^{\prime}, \mathbf{q}_{j}^{\prime}\right)=e^{-\left(\mu \frac{\left(\mathbf{x}_{i}^{\prime}-\mathbf{x}_{j}^{\prime}\right) \cdot\left(\mathbf{x}_{i}-\mathbf{x}_{j}\right)}{\left\|\mathbf{x}_{i}^{\prime}-\mathbf{x}_{j}^{\prime}\right\|\left\|\mathbf{x}_{i}-\mathbf{x}_{j}\right\|}+\nu \frac{\|\| \mathbf{x}_{i}^{\prime}-\mathbf{x}_{j}^{\prime}\|-\| \mathbf{x}_{i}-\mathbf{x}_{j}\|\| \|}{\left\|\mathbf{x}_{i}-\mathbf{x}_{j}\right\|}\right.}
$$

The potential is set so that the global shape of the model will be kept by penalizing the deviation of the angle and distance between vertices from our model.

The local observation is defined as

$$
B_{i}\left(\mathbf{q}_{i}^{\prime}\right)=e^{\xi\left\|\mathbf{g}_{i}^{\prime} \cdot \mathbf{g}_{i}\right\|}
$$

which means to penalize candidates with weak gradient amplitude and inconsistent gradient direction with the model.

Under these definitions, a bone contour that keeps the global shape of our model and at the same time locates itself to the strong edge positions can be obtained by a Maximal Likelihood(ML) estimation as

$$
C_{\text {image }}^{*}=\max _{\mathbf{Q}_{\text {cand }}=\left\{\mathbf{q}_{i}^{\prime}\right\}} \prod_{i=0}^{M-1} B_{i}\left(\mathbf{q}_{i}^{\prime}\right) \prod_{i, j, e_{i, j=1}} U_{i, j}\left(\mathbf{q}_{i}^{\prime}, \mathbf{q}_{j}^{\prime}\right)
$$

In our approach, the candidate positions for each node of the bone contour are sampled along the normal direction of the model and standard loopy belief propagation[21] is used to approximate the ML estimation and an example of the method is shown in Fig. 4.

\subsubsection{D/3D nonrigid registration}

Our statistical model can be fitted to the extracted bone contours $\left\{C_{\text {image }}^{k, n}\right\}$ as a $2 \mathrm{D} / 3 \mathrm{D}$ nonrigid registration procedure. The method described in [8] is applied here as follows. For each point $P_{l}$ on the extracted bone contour, the correspondence between its backprojection line $B P\left(P_{l}\right)$ and a vertex $v_{\operatorname{corr}\left(P_{l}\right)}$ on the current instanced statistical model $\mathcal{M}: \mathbf{S}\left(\mathbf{Q}^{n}\right)$ and its current transformation $\mathbf{T}^{n}$ can be established. Project $v_{\operatorname{corr}\left(P_{l}\right)}$ on $B P\left(P_{l}\right)$ will generate a correspondent $3 \mathrm{D}$ point pair $\left(v_{\operatorname{corr}\left(P_{l}\right)}, \operatorname{Proj}\left(v_{\operatorname{corr}\left(P_{l}\right)}, B P\left(P_{l}\right)\right)\right)$. A rigid transformation $\mathbf{T}_{\text {update }}^{n+1}$ can be calculated to align the current statistical model $\mathcal{M}: \mathbf{S}\left(\mathbf{Q}^{n}\right)$ to the extracted contours. The rigid 


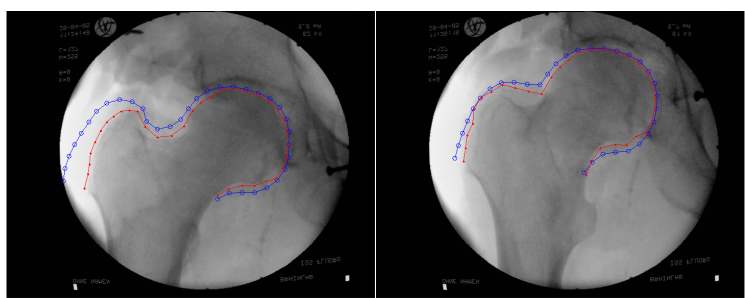

Figure 4. Bayesian network based 2D segmentation, where circles show the projected silhouettes and dots show the extracted contours

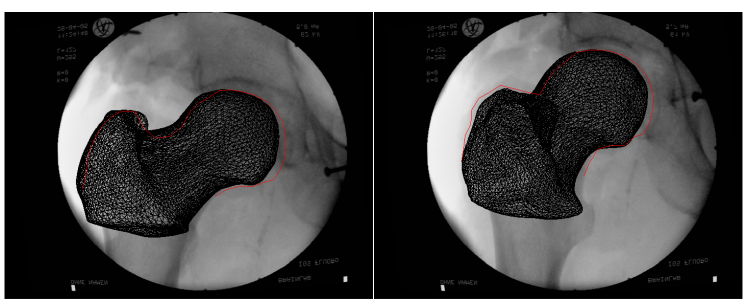

Figure 5. 2D/3D nonrigid registration

transformation can then be updated as $\mathbf{T}^{n+1}=\mathbf{T}_{\text {update }}^{n+1} \mathbf{T}^{n}$. The residual error between correspondent point pairs can then be compensated by the constrained deformation of the statistical model [16]. An example of the nonrigid registration is shown in Fig. 5.

\section{Experimental Results}

We verified our approach on three set of clinical data, each data set includes four calibrated fluoroscopic images of the proximal femur. To test the robustness of the automatic initialization, we run the initialization algorithm for 10 trails on each data set with particle number $N=200$. In each trial the proximal femur is correctly identified and the statistical results are shown in Table 1. The extracted proximal femur contours are shown in Fig. 6.

\section{Conclusions}

In this paper we propose a 3D statistical model based fully automatic bone contour extraction framework from calibrated fluoroscopic images. We solve the automatic initialization by fitting a simplified multiple component geometrical 3D model to the observed fluoroscopic images. The 3D model based initialization algorithm does not ask for strict view direction assumption compared with 2D
Table 1. Statistical results of the automatic initialization algorithm, all results are relative to the mean values of the 10 trials

\begin{tabular}{|l|c|c|c|}
\hline Parameter & Data Set 1 & Data Set 2 & Data Set 3 \\
\hline Head Center (mm) & $1.4 \pm 1.1$ & $0.1 \pm 0.1$ & $0.1 \pm 0.2$ \\
\hline Head Radius (mm) & $0.3 \pm 0.4$ & $0.6 \pm 0.2$ & $1.0 \pm 0.8$ \\
\hline Neck Radius (mm) & $0.8 \pm 1.1$ & $0.6 \pm 0.9$ & $1.0 \pm 1.2$ \\
\hline Neck Length (mm) & $1.0 \pm 1.4$ & $1.3 \pm 1.8$ & $1.2 \pm 1.7$ \\
\hline Neck Axis (degree) & $0.8 \pm 0.7$ & $2.3 \pm 1.0$ & $1.8 \pm 1.1$ \\
\hline Shaft Radius(mm) & $0.2 \pm 0.3$ & $0.1 \pm 0.2$ & $0.2 \pm 0.2$ \\
\hline Shaft Length(mm) & $0.5 \pm 0.2$ & $0.9 \pm 0.5$ & $1.8 \pm 1.0$ \\
\hline Neck/Shaft Angle(degree) & $0.8 \pm 1.0$ & $2.0 \pm 2.5$ & $1.8 \pm 2.6$ \\
\hline
\end{tabular}

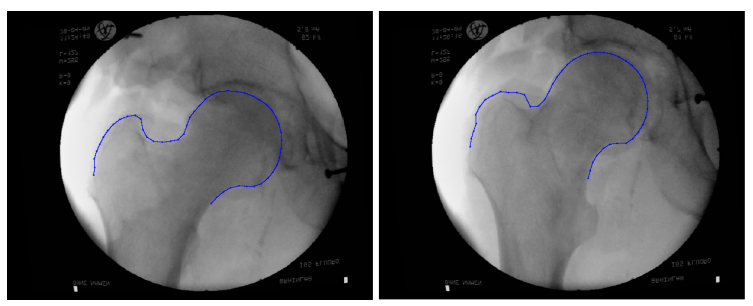

(a) Data set 1

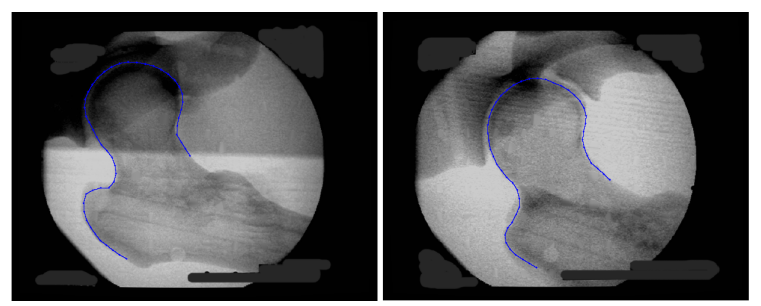

(b) Data set 2

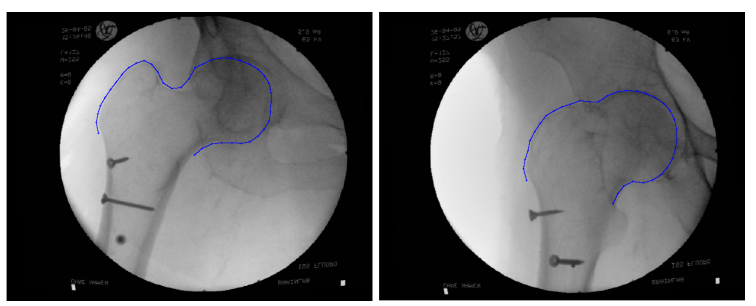

(c) Data set 3

Figure 6. Results of automatic proximal femur bone contour extraction on clinical data 
model or 2D image feature based initialization. Since the fitting is accomplished by a particle filter based evolutionary algorithm, it has a strong capability to overcome local optima and converge to the global optimal. The 3D statistical model based bone contour extraction is solved as a simultaneous 2D/3D registration and segmentation. The model based segmentation is accomplished by a Bayesian inference procedure which in principle can overperform active contour and AAM/ASM by simultaneously optimize both the global shape constraints and local image feature information. Experiments on clinical data sets verified the validity and performance of this approach.

\section{References}

[1] Y. Chen, X. H. Ee, W. K. Leow, and T. S. Howe, "Automatic Extraction of Femur Contours from Hip X-ray Images," CVBIA'05, LNCS 3765, pp. 200-209, 2005.

[2] R. de Luis-Garcia, M. Martin-Fernadez, J. I. Arribas, and C. Alberola-Lopez, "A Fully Automatic Algorithm for Contour Detection of Bones in Hand Radiographs Using Active Contours," ICIP03, Part. III, pp. 421-424, 2003.

[3] M. G. Roberts, T. F. Cootes, and J. E. Adams, "Automatic segmentation of lumbar vertebrae on digitised radiographs using linked active appearance models," MIUA'06, Part. I, pp. 120124, 2006.

[4] H. Gottschling, M. Roth, A. Schweikard, and R. Burgkart, "Intraoperative, Fluoroscopy-based Planning for Complex Osteotomies of the Proximal Femur," The International Journal of Medical Robotics \& Computer Assisted Srugery, Vol. 1(3), pp. 67-73, 2005.

[5] F. Bartolini, M. Carfagni, L. Governi, "Model-based Extraction of Femoral Medulla Ducts from Radiographic Images," Image and Vision Computing, Vol. 22, pp. 173-182, 2004.

[6] T. P. Tian, Y. Chen, W. K. Leow, W. Hsu, T. S. Howe, and M. A. Png, "Computing Neck-Shaft Angle of Femur for X-ray Fracture Detection,” CAIP'03, LNCS 2756, pp. 82-89, 2003.

[7] S. Benameur, M. Mignotte, S. Parent, H. Labelle, W. Skalli, and J. A. de Guise "3D/2D Registration and Segmentation of Scoliotic Vertebrae Using Statistical Models," Computerized Medical Imaging and Graphics, Vol. 27(5), pp. 321-337, 2003.

[8] G. Zheng and L.-P. Nolte, "Surface Reconstruction of Bone from X-ray Images and Point Distribution Model Incorporating a Novel Method for 2D-3D Correspondence," CVPR'06, Part II., pp. 2237-2244, 2006.

[9] H. Lamecker, T. H. Wenckebach, H. C. Hege, "Atlas-based 3D-Shape Reconstruction from X-ray Images,' ICPR'06, Part I., pp. 371-374, 2006.
[10] T. S. Tang, and R. E. Ellis, “2D/3D Deformable Registration Using a Hybrid Atlas,' MICCAI'05, Part II., LNCS 3750, pp. 223-230, 2006.

[11] G. Behiels, D. Van der Meulen, F. Maes, P. Suetens, and P. Dewaele, "Active Shape Model-based Segmentation of Digital X-ray Images,” MICCAI'99, LNCS 1679, pp. 128-137, 1999.

[12] B. Howe, A. Gururajan, and L. R. Long, "Hierarchical Segmentation of Cervical and Lumbar Vertebrae Using a Customised Generalized Hough Transform and Extensions to Active Appearance Models," Proc. IEEE 6th SSIAI, pp. 182-186, March 2004.

[13] G. Langs, P. Peloschek, and H. Bischof, "Determining Position and Fine Shape Detail in Radiological Anatomy," Pattern Recognition, Proc. of 25th DAGM Symposium, LNCS 2781, pp. 532-539, 2003.

[14] M. Seise, S. J. McKenna, I. W. Ricketts, and C. A. Wigderowitz,"Probabilistic Segmentation of the Knee Joint from X-ray Images," MIUA'06, pp. 110-114, 2006.

[15] M. de Bruijne, M. Nielsen, "Image Segmentation by Shape Particle Filtering," ICPR'04, Part. III, 2004.

[16] G. Zheng, K. T. Rajamani, L.-P. Nolte, "Use of a Dense Surface Point Distribution Model in a Three-Stage Anatomical Shape Reconstruction from Sparse Information for Computer Assisted Orthopaedic Surgery: A Preliminary Study," ACCV'06, Part. II, LNCS 3852, pp. 52-60, 2006.

[17] B. Ma, and R. E. Ellis, "Surface-based Registration with a Particle Filter," MICCAI'04, Part I. LNCS 3216, pp. 566-573, 2004.

[18] M. W. Lee, and I. Cohen, "Human Upper Body Pose Estimation in Static Images," ECCV'04, Part II., LNCS 3022, pp. 126-138, 2004.

[19] L. Sigal, S. Bhatia, S. Roth, M. J. Black, and M. Isard, "Tracking loose-limbed people," CVPR'04, Part. 1, pp. 421428, 2004.

[20] Y. Wu, G. Hua and T. Yu, "Tracking articulated body by dynamic Markov network,' ICCV'03, vol. 1, pp. 1094-1101, 2003.

[21] J. Coughlan, S. Ferreira, "Finding deformable shapes using loopy belief propagation," ECCV'02, Part III., pp. 453-468, 2002.

[22] A. Rangarajan, J. Coughlan, and A. L. Yuille, "A Bayesian Network Framework for Relational Shape Matching," ICCV'03, pp. 671-678, 2003.

[23] E. B. Sudderth, M. I. Mandel, W. T. Freeman, and A. S. Willsky, "Visual Hand Tracing Using Nonparametric Belief Propagation," IEEE CVPR Workshop on Generative Model Based Vision, 2004. 